University of Nebraska - Lincoln

DigitalCommons@University of Nebraska - Lincoln

2001

Building Blocks for Reliable Complex Nonlinear Numerical

Simulations

Helen Yee

Follow this and additional works at: https://digitalcommons.unl.edu/nasapub

Part of the Astrophysics and Astronomy Commons

This Article is brought to you for free and open access by the National Aeronautics and Space Administration at DigitalCommons@University of Nebraska - Lincoln. It has been accepted for inclusion in NASA Publications by an authorized administrator of DigitalCommons@University of Nebraska - Lincoln. 


\title{
BUILDING BLOCKS FOR RELIABLE COMPLEX NONLINEAR NUMERICAL SIMULATIONS*
}

\author{
H. C. Yee \\ NASA Ames Research Center, Moffett Field, CA 94035, USA. \\ yee@nas.nasa.gov
}

\begin{abstract}
This chapter describes some of the building blocks to ensure a higher level of confidence in the predictability and reliability (PAR) of numerical simulation of multiscale complex nonlinear problems. The focus is on relating PAR of numerical simulations with complex nonlinear phenomena of numerics. To isolate sources of numerical uncertainties, the possible discrepancy between the chosen partial differential equation (PDE) model and the real physics and/or experimental data is set aside. The discussion is restricted to how well numerical schemes can mimic the solution behavior of the underlying PDE model for finite time steps and grid spacings. The situation is complicated by the fact that the available theory for the understanding of nonlinear behavior of numerics is not at a stage to fully analyze the nonlinear Euler and Navier-Stokes equations. The discussion is based on the knowledge gained for nonlinear model problems with known analytical solutions to identify and explain the possible sources and remedies of numerical uncertainties in practical computations. Examples relevant to turbulent flow computations are included.
\end{abstract}

Keywords: Dynamics of Numerics, Chaotic Transients, Direct Numerical Simulations, Reliability of Numerical Simulations, Under-Resolved Grids, Spurious Numerical Solutions, Nonlinear Dynamics, Spurious Bifurcations.

\section{Introduction}

The last two decades have been an era when computation is ahead of analysis and when very large scale practical computations are increasingly used in poorly understood multiscale complex nonlinear physical problems and non-traditional fields. Ensuring a higher level of confidence in the predictability and reliability (PAR) of these numerical simulations could play a major role in furthering the design, understanding, affordability and safety of our next generation air

${ }^{*}$ A chapter for Turbulent Flow Computation, (Eds. D. Drikakis \& B. Geurts), Kluwer Academic Publisher, 2001. 
and space transportation systems, and systems for planetary and atmospheric sciences, and astrobiology research. In particular, it plays a major role in the success of the US Accelerated Strategic Computing Initiative (ASCI) and its five Academic Strategic Alliance Program (ASAP) centers. Stochasticity stands alongside nonlinearity and the presence of multiscale physical processes as a predominant feature of the scope of this research. The need to guarantee PAR becomes acute when computations offer the ONLY way of generating this type of data limited simulations, the experimental means being unfeasible for any of a number of possible reasons. Examples of this type of data limited problem are:

- Stability behavior of re-entry vehicles at high speeds and flow conditions beyond the operating ranges of existing wind tunnels

- Flow field in thermo-chemical nonequilibrium around space vehicles traveling at hypersonic velocities through the atmosphere (lack sufficient experimental or analytic validation)

- Aerodynamics of aircraft in time-dependent maneuvers at high angles of attack (free of interference from support structures, wind-tunnel walls etc., and able to treat flight at extreme and unsafe operating conditions)

- Stability issues of unsteady separated flows in the absence of all the unwanted disturbances typical of wind-tunnel experiments (e.g., geometrically imperfect free-stream turbulence)

This chapter describes some of the building blocks to ensure a higher level of confidence in the PAR of numerical simulation of the aforementioned multiscale complex nonlinear problems, especially the related turbulence flow computations. To isolate the source of numerical uncertainties, the possible discrepancy between the chosen model and the real physics and/or experimental data is set aside for the moment. We concentrate only on how well numerical schemes can mimic the solution behavior of the underlying partial different equations (PDEs) for finite time steps and grid spacings. Even with this restriction, the study of PAR encompasses elements and factors far beyond what is discussed here. It is important to have a very clear distinction of numerical uncertainties from each source. These include but are not limited to (a) stability and well-posedness of the governing PDEs, (b) type, order of accuracy, nonlinear stability, and convergence of finite discretizations, (c) limits and barriers of existing finite discretizations for highly nonlinear stiff problems with source terms and forcing, and/or for long time wave propagation phenomena, (d) numerical boundary condition (BC) treatments, (e) finite representation of infinite domains, (f) solution strategies in solving the nonlinear discretized equations, $(\mathrm{g})$ procedures for 
obtaining the steady-state numerical solutions, (h) grid quality and grid adaptations, (i) multigrids, and (j) domain decomposition (zonal or multicomponent approach) in solving large problems. At present, some of the numerical uncertainties can be explained and minimized by traditional numerical analysis and standard CFD practices. However, such practices, usually based on linearized analysis, might not be sufficient for strongly nonlinear and/or stiff problems. We need a good understanding of the nonlinear behavior of numerical schemes being used as an integral part of code verification, validation and certification.

A major stumbling block in genuinely nonlinear studies is that unlike the linear model equations used for conventional stability and accuracy considerations in time-dependent PDEs, there is no equivalent unique nonlinear model equation for nonlinear hyperbolic and parabolic PDEs for fluid dynamics. On one hand, a numerical method behaving in a certain way for a particular nonlinear differential equation (DE) (PDE or ordinary differential equation (ODE)) might exhibit a different behavior for a different nonlinear DE even though the DEs are of the same type. On the other hand, even for simple nonlinear model DEs with known solutions, the discretized counterparts can be extremely complex, depending on the numerical methods. Except in special cases, there is no general theory at the present time to characterize the various nonlinear behaviors of the underlying discretized counterparts. Herein, the discussion is based on the knowledge gained for nonlinear model problems with known analytical solutions to identify and explain the possible sources and remedies of numerical uncertainties in practical computations.

The term "discretized counterparts" is used to mean the finite difference equations resulting from finite discretizations of the underlying DEs. Here "dynamics" is used loosely to mean the dynamical behavior of nonlinear dynamical systems (continuum or discrete) and "numerics" is used loosely to mean the numerical methods and procedures in solving dynamical systems. We emphasize here that in the study of the dynamics of numerics, unless otherwise stated, we always assume the continuum (governing equations) is nonlinear.

Outline: Section 2 discusses the sources of nonlinearities and the knowledge gained from studying the dynamics of numerics for nonlinear model problems. Sections 3-5 discuss some of the relevant issues and building blocks for a more reliable (and predictability) numerical simulation in more details. Section 6 shows examples of spurious numerics relevant to turbulent flow computations. 


\section{Sources of Nonlinearities and Knowledge Gained from Nonlinear Model Problems}

Two of the building blocks for the PAR of numerical simulations are to identify all the sources of nonlinearities and to isolate the elements and issues of numerical uncertainties due to these nonlinearities.

Sources of Nonlinearities: The sources of nonlinearities that are well known in computational fluid dynamics (CFD) are due to the physics. Examples of nonlinearities due to the physics are convection, diffusion, forcing, turbulence source terms, reacting flows, combustion related problems, or any combination of the above. The less familiar sources of nonlinearities are due to the numerics. There are generally three major sources:

- Nonlinearities due to time discretizations - the discretized counterpart is nonlinear in the time step. Examples of this type are Runge-Kutta methods. If fixed time steps are used, spurious steady-state or spurious asymptotic numerical solutions can occur, depending on the the initial condition (IC). Linear multistep methods (LMMs) (Butcher 1987) are linear in the time step, and they do not exhibit spurious steady states. See Yee \& Sweby (1991-1997) and references cited therein for the dynamics of numerics of standard time discretizations.

- Nonlinearities due to spatial discretizations - in this case, the discretized counterpart can be nonlinear in the grid spacing and/or the scheme. Examples of nonlinear schemes are the total variation diminishing (TVD), essentially nonoscillatory (ENO) and weighted ENO (WENO) schemes. The resulting discretized counterparts are nonlinear (in the dependent variables) even though the governing equation is linear. See Yee (1989) and references cited therein for the forms of these schemes.

- Nonlinearities due to complex geometries, boundary interfaces, grid generation, grid refinements and grid adaptations (Yee \& Sweby 1995) - each of these procedures can introduce nonlinearities even though the governing equation is linear.

Continuous and Discrete Dynamical Systems: Before analyzing the dynamics of numerics, it is necessary to analyze (or understand) as much as possible the dynamical behavior of the governing equations and/or the physical problems using theories of DEs, dynamical systems of DEs, and also physical guidelines. For stability and well-posedness considerations, whenever it is possible, it is also necessary to condition (not pre-condition) the governing PDEs before the application of the appropriate scheme (Yee \& Sjögreen 2001a,b). The discretized counterparts are dynamical systems on their own. They have their own dynamics, and they are different from one numerical method to another in space 
and time and are different from the underlying governing PDE (Yee \& Sweby 1993). The procedures of solving the nonlinear algebraic systems resulting from using implicit methods can interfere with or superpose unwanted behavior on the underlying scheme. Also, the same scheme can exhibit different spurious behavior when used for time-accurate vs. time marching to the steady states. For a combination of initial condition and time step, a super-stable scheme can stabilize unstable physical (analytic) steady states (Yee \& Sweby 1993-1996). Super-stable scheme here refers to the region of numerical stability enclosing the physical instability of the true solution of the governing equation. Yee et al. and Yee \& Sweby (1991-1997) divide their studies into two categories, steady state and time accurate computations. Within each category they further divide the governing PDEs into homogeneous and nonhomogeneous (i.e., with or without source terms), and rapidly/slowly developing and long time integration problems.

Knowledge Gained from Nonlinear Model Problems: With the aid of elementary examples, Yee et al., Yee \& Sweby (1991-1997), Sweby \& Yee (19941995) and Griffiths et al. (1992a,b) discuss the fundamentals of spurious behavior of commonly used time and spatial discretizations in CFD. Details of these examples can be found in their earlier papers. These examples consist of nonlinear model ODEs and PDEs with known analytical solutions (the most straight forward way of being sure what is "really" happening with the numerics). They illustrate the danger of employing fixed (constant) time steps and grid spacings. They were selected to illustrate the following different nonlinear behavior of numerical methods:

- Occurrence of stable and unstable spurious asymptotes above the linearized stability limit of the scheme (for constant time steps)

- Occurrence of stable and unstable spurious steady states below the linearized stability limit of the scheme (for constant time steps)

- Stabilization of unstable steady states by implicit and semi-implicit methods

- Interplay of initial data and time steps on the occurrence of spurious asymptotes

- Interference with the dynamics of the underlying implicit scheme by procedures in solving the nonlinear algebraic equations (resulting from implicit discretizations of the continuum equations)

- Dynamics of the linearized implicit Euler scheme solving the time-dependent equations to obtain steady states vs. Newton's method solving the steady equation 
- Spurious dynamics independently introduced by spatial and time discretizations

- Convergence problems and spurious behavior of high-resolution shockcapturing methods

- Numerically induced \& suppressed (spurious) chaos, and numerically induced chaotic transients

- Spurious dynamics generated by grid adaptations

Here "spurious numerical solutions (and asymptotes)" is used to mean numerical solutions (asymptotes) that are solutions (asymptotes) of the discretized counterparts but are not solutions (asymptotes) of the underlying DEs. Asymptotic solutions here include steady-state solutions, periodic solutions, limit cycles, chaos and strange attractors. See Thompson \& Stewart (1986) and Hoppensteadt (1993) for the definition of chaos and strange attractors.

\section{Minimization of Spurious Steady State via Bifurcation Theory}

The use of time-marching approaches to obtain steady-state numerical solutions has been considered the method of choice in computational physics for three decades since the pioneering work of Moretti \& Abbett (1966). Moretti and Abbett used this approach to solve the inviscid supersonic flow over a blunt body without resorting to solving the steady form of PDEs of the mixed type. Much success was achieved in computing a variety of weakly and moderately nonlinear fluid flow problems. For highly complex nonlinear problems, the situation is more complicated. The following isolates some of the key elements and issues of numerical uncertainties in time-marching to the steady state. Studies in Yee et al. (1991-1997) indicate that each of the following can affect not only the convergence rate but also spurious numerics other than standard stability and accuracy linearized numerical analysis problems.

- Solving an initial boundary value problem (IBVP) with unknown initial data

- Reliability of residual test

- Methods used to accelerate the convergence process

- Precondition (not condition) the governing PDE (might introduce additional spurious solutions beyond the solution of the underlying PDE)

- Precondition the discretized counterparts (might introduce additional spurious solutions beyond the underlying discretized system) 
- Methods in solving the nonlinear algebraic equations from implicit methods

- Mismatch in implicit schemes

- Nonlinear schemes

- Schemes that are linear vs. nonlinear in time step

- Adaptive time step based on local error control

It is a standard practice in time-marching to the steady-state numerical solutions to use "local time step" (varied from grid point to grid point using the same CFL) for nonuniform grids. However, except in finite element methods, an adaptive time step based on local error control is rarely used. An adaptive time step is built in for standard ODE solver computer packages. It enjoyed much success in controlling accuracy and stability for transient (time-accurate) computations. The issue is to what extent this adaptive local error control confers global properties in long time integration of time-dependent PDEs and whether one can construct a similar error control that has guaranteed and rapid convergence to the correct steady-state numerical solutions in the time-marching approaches for time-dependent PDEs.

One can see that the construction of adaptive time integrators for timemarching to the steady states demands new concepts and guidelines and is distinctively different than for the time-accurate case. Straightforward application of adaptive time integrators for time-accurate computations might be inappropriate and/or extremely inefficient for time-marching to the steady state. For example, an adaptive time step based on local error control for accuracy considerations is irrelevant before a steady state has been reached. Moreover, this type of local error control might hinder the speed of the convergence process with no guarantee of leading to the correct steady state.

The twin requirements of guaranteed and rapid convergence to the correct steady-state numerical solution are most often conflicting, and require a full understanding of the global nonlinear behavior of the numerical scheme as a function of the discretized parameters, grid adaptation parameters, initial data and boundary conditions. We believe tools from bifurcation theory can help to minimize spurious steady-state numerical solutions.

In many fluid problems the solution behavior is well known for certain values of the physical parameters but unknown for other values. For these other values of the parameters, the problem might become very stiff and/or strongly nonlinear, making the available numerical schemes (or the scheme in use) intractable. In this situation, continuation methods in bifurcation theory can become very useful. If possible, one should start with the physical parameter of a known or 
reliable steady state (e.g., flow behavior is usually known for low angles of attack but not for high angles of attack). One can then use a continuation method such as the improved pseudo arclength continuation method of Keller (1977) (or the recent developments in this area) to solve for the bifurcation curve as a function of the physical parameter. See e.g., Doedel (2000), Shroff \& Keller (1993) and Davidson (1997). The equations used are the discretized counterpart of the steady PDEs or the time-dependent PDEs. See Stephen \& Shubin (1981) and Shubin et al. (1981) for earlier related work. If time-marching approaches are used, a reliable steady-state numerical solution (as a starting value on the correct branch of the bifurcation curve for a particular value of the physical parameter) is assumed. This starting steady-state numerical solution is assumed to have the proper time step and initial data combination and to have a grid spacing fine enough to resolve the flow feature. The continuation method will produce a continuous spectrum of the numerical solutions as the underlying physical parameter is varied until it arrives at a critical value $p_{c}$ such that it either experiences a bifurcation point or fails to converge. Since we started on the correct branch of the bifurcation curve, the solution obtained before that $p_{c}$ should be more reliable than if one starts with the physical parameter in question with unknown initial data and tries to stretch the limitation of the scheme. Note that by starting a reliable solution on the correct branch of the bifurcation curve, the dependence of the numerical solution on the initial data associated with time-marching methods can be avoided before a spurious bifurcation occurs.

Finally, when one is not sure of the numerical solution, the continuation method can be used to double check it. This approach can even reveal the true limitations of the existing scheme. In other words, the approach can reveal the critical physical parameter for which the numerical method breaks down. On the other hand, if one wants to find out the largest possible time step and/or grid spacing that one can use for a particular problem and physical parameter, one can also use continuation methods to trace out the bifurcation curve as a function of the time step and/or grid spacing. In this case, one can start with a small time step and/or grid spacing with the correct steady state and observe the critical discretized parameter as it undergoes instability or spurious bifurcation. Of course, this method for minimizing spurious steady states still can suffer from spurious behavior due to an under-resolved grid because of limited computer resources for complex practical problems. Practical guidelines to avoid under-resolved grids are yet another important building block toward reliable numerical simulations. The efficient treatment of solving the extremely large set of eigenvalue problems to study the type and stability of bifurcation points is yet another challenge. See, e.g., Fortin et al. (1996), Davidson (1997) and Shroff \& Keller (1993) for some discussions. Consequently, further develop- 
ment in numerical bifurcation analysis and new concepts in adaptive methods for time-marching to steady state hold a key to the minimization of spurious numerics.

\section{Source Term Treatments in Reacting Flows}

In the modeling of problems containing finite-rate chemistry or combustion, often, a wide range of space and time scales is present due to the reacting terms, over and above the different scales associated with turbulent flows, leading to additional numerical difficulties. This stems mainly from the fact that the majority of widely used numerical algorithms in reacting flows were originally designed to solve non-reacting fluid flow problems. Fundamental studies on the behavior of these schemes for reacting model problems by the author and collaborators were reported in Yee \& Sweby (1997) and references cited therein. In a majority of these studies, theory from dynamical systems was used to gain a better understanding of the nonlinear effects on the performance of these schemes. The main findings are:

- It was shown in LeVeque and Yee (1990) that, for stiff reactions containing shock waves, it is possible to obtain stable solutions that look reasonable and yet are completely wrong, because the discontinuities are in the wrong locations. Stiff reaction waves move at nonphysical wave speeds, often at the rate of one grid cell per time step, regardless of their proper speed. There exist several methods that can overcome this difficulty for a single reaction term. For more than a single reacting term in fully coupled nonlinear systems, more research is needed. One impractical way of minimizing the wrong speed of propagation of discontinuities is to demand orders of magnitude grid size reduction compared with what appears to be a reasonable grid spacing in practice.

- It was shown in Lafon and Yee $(1991,1992)$ that the numerical phenomenon of incorrect propagation speeds of discontinuities may be linked to the existence of some stable spurious steady-state numerical solutions.

- It was also shown in Lafon and Yee $(1991,1992)$ that various ways of discretizing the reaction term can affect the stability of and convergence to the spurious numerical steady states and/or the exact steady states. Pointwise evaluation of the source terms appears to be the least stable.

- It was shown in Yee et al. (1991) and Griffiths et al. (1992a,b) that spurious discrete traveling waves can exist, depending on the method of discretizing the source term. When physical diffusion is added, it is not known what type of numerical difficulties will surface. 
From the above findings we can safely conclude that understanding the nonlinear behavior of numerical schemes for reacting flows and the effects of finite-rate chemistry on turbulence is in its infancy. However, we believe that knowledge gained from fundamental studies is helpful to improve some of the numerical difficulties that were encountered in the past.

\section{Adaptive Numerical Methods}

Another important building block for PAR is adaptive numerical methods. This includes adaptive temporal and spatial schemes, grid adaptation as an integral part of the numerical solution process, and, most of all, adaptive numerical dissipation controls. Using tools from dynamical systems, Yee et al. (1991-1997), Yee \& Sweby (1993-1997), Griffiths et al. (1992a,b) and Lafon \& Yee $(1991,1992)$ showed that adaptive temporal and adaptive spatial schemes are important in minimizing numerically induced chaos, numerically induced chaotic transients and the false prediction of flow instability by direct numerical simulation (DNS). Their studies further indicate the need in the development of practical adaptive temporal schemes based on error controls to minimize spurious numerics due to the full discretizations. In addition, the development of adaptive temporal and spatial schemes based on error controls to minimize numerical artifacts due to the full discretizations is also needed. This is due to the fact that adaptive temporal or adaptive spatial schemes alone will not be able to provide an accurate and reliable process to minimize numerical artifacts for time-accurate computations. Guided by the theory of nonlinear dynamics, Yee et al. (1997) and Yee \& Sweby (1997) presented practical examples which illustrated the danger of using nonadaptive temporal and spatial schemes for studying flow instability.

On the subject of adaptive numerical dissipation controls, it is well known that reliable, accurate and efficient direct numerical simulation (DNS) of turbulence in the presence of shock waves represents a significant challenge for numerical methods. Standard TVD, ENO, WENO and discontinuous Galerkin types of shock-capturing methods for the Euler equations are now routinely used in high speed blast wave simulations with virtually non-oscillatory, crisp resolution of discontinuities (see reference section). For the unaveraged unsteady compressible Navier-Stokes equations, it was observed that these schemes are still too dissipative for turbulence and transition predictions. On the other hand, hybrid schemes, where spectral and/or higher-order compact (Padé) schemes are switched to higher-order ENO schemes when shock waves are detected, have their deficiencies. One shortcoming of this type of hybridization is that the numerical solution might experience a non-smooth transition at the switch to a different type of scheme. For 2-D and 3-D complex shock wave and shear 
surface interactions, the switch mechanism can become non-trivial and frequent activation of shock-capturing schemes is possible.

The recent work of Yee et al. (1999, 2000), Sjögreen \& Yee $(2000,2001)$, and Yee \& Sjögreen $(2001 \mathrm{a}, \mathrm{b})$ indicates that appropriate adaptive numerical dissipation control is essential to control nonlinear instability in general, and for long time integration, in particular. An integrated design approach on the construction of adaptive numerical dissipation controls can be found in Yee \& Sjögreen $(2001 \mathrm{a}, \mathrm{b})$.

\section{Spurious Numerics Relevant to Turbulent Flow Computations}

This section illustrates four numerical examples that exhibit spurious numerics relevant to turbulent flow computations. The first example discusses spurious vortices related to under-resolved grids and/or lack of appropriate numerical dissipation/filter controls. The second example discusses spurious behavior of super-stable implicit time integrators. The last two examples discuss spurious behavior near the onset of turbulence and/or the onset of instability of the steady state solution. If care is not taken, spurious bifurcation of the discretized counterpart and/or a numerically induced chaotic transient can be mistaken for the onset of physical turbulence of the governing equation. These examples can serve to illustrate the connection between the spurious numerical phenomena observed in simple nonlinear models and CFD computations.

\subsection{Spurious Vortices in Under-Resolved Incompressible Thin Shear Layer Flow Simulations}

Brown \& Minion (1995) performed a thorough study of a second-order Godunov-projection method and a fourth-order central difference method for the 2-D incompressible Navier-Stokes equations, varying the resolution of the computational mesh with the rest of the physical and discretization parameters fixed. This is a good example of isolating the cause of spurious behavior. The physical problem is a doubly periodic double shear layer. The shear layers are perturbed slightly at the initial time, which causes the shear layer to roll up in time into large vortical structures. For a chosen shear layer width that is considered to be thin and a fixed perturbation size, they compared the solution for four different grid sizes $(64 \times 64,128 \times 128,256 \times 256,512 \times 512)$ with a reference solution using a grid size of $1024 \times 1024$. For the $256 \times 256$ grid, a spurious vortex was formed midway between the periodically repeating main vortex on each shear layer. The $128 \times 128$ solution showed three spurious vortices along the shear layer. The spurious vortex disappeared with a $512 \times 512$ mesh. They also disabled the flux limiters (a strictly upwind Fromm's 
method), and found the behavior to be similar. A subsequent study (Minion $\&$ Brown 1997) using five different formulations and six different commonly used schemes in CFD found similar behavior. They concluded that the spurious vortex is the artifact of an under-resolved grid and the behavior is caused by a nonlinear effect. Linking this behavior with nonlinear dynamics, we interpret their observation as follows. For the particular grid size and time step combination, stable spurious equilibrium points were introduced by the numerics into a portion of the flow field while the major portion of the flow field was predicted correctly. In other words, the spurious vortices are the solution of the discretized counterpart for that particular range of grid size and time step. The number of stable spurious vortices is a function of the grid size. As the grid spacing decreases, the spurious equilibria gradually become unstable and the numerical solution mimics the true solution.

Instead of merely increasing the grid size, there are situations where underresolved grids can be overcome by proper control of numerical dissipation/filters. It was shown in Fischer \& Mullen that high-order spectral element methods (Maday \& Patera 1989), coupled with filter-based dissipation, can remove what is believed to be an underresolution-induced spurious vortex numerical solution. See Fischer \& Mullen or Yee \& Sjögreen (2001a,b) for a discussion. Fischer \& Mullen or Yee \& Sjögreen (2001a,b) illustrate the added benefit of adaptive numerical dissipation/filter controls for high order or high resolution shock-capturing schemes.

\subsection{Stabilizing Unstable Steady States with Implicit Time Integrators (Poliashenko \& Yee 1995, unpublished)}

This is a joint work with Maxim Poliashenko in 1995. This unpublished work was presented at the 10th International Conference in Finite Element Methods, January 5-8, 1998, Tucson, Arizona, and also has been presented at various invited lectures during the last four years. We consider a 2-D lid driven cavity (LDC) problem. The PDE governing equations are the ideal viscous incompressible Navier-Stokes equations of the form

$$
\begin{aligned}
& \mathbf{u}_{t}+\left(\mathbf{u}^{T} \nabla\right) \mathbf{u}=-\nabla p+\frac{1}{R e} \triangle \mathbf{u}, \\
& \operatorname{div} \mathbf{u}=0,
\end{aligned}
$$

with boundary conditions in the domain $(x, y)$

$$
\begin{aligned}
\mathbf{u}(y=a) & =1, \\
\mathbf{u}(y=0)=\mathbf{u}(x=0) & =\mathbf{u}(x=1)=0 .
\end{aligned}
$$

Here $\mathbf{u}$ is a 2-D velocity vector, $p$ is pressure and $R e$ is the Reynolds number, a dimensionless parameter of the problem that describes the relationship between 
kinematic and viscous forces in the fluid. Here $a$ is the cavity aspect ratio. The velocity vector $\mathbf{u}$, pressure $p$ and the time $t$ are normalized with $R e$ being proportional to the velocity of the lid and inversely proportional to the viscosity of the fluid.

Several numerical time integrators indicated that while the steady state is unique and stable for small Reynolds numbers, the flow becomes time-periodic as $R e$ is increased to a few thousand. Poliashenko and Aidun (1995) applied their direct method for computations of co-dimension one bifurcations to show that the steady state of the LDC problem indeed loses its stability via Hopf bifurcation (Thompson \& Stewart 1986) as the Reynolds number increases, giving rise to a time-periodic solution. This bifurcation can be subcritical or supercritical depending on the aspect ratio $a$. With a given spatial resolution, they found that the Hopf bifurcation point is supercritical for $a=0.8$ at $R e=$ 5220 , and for $a=1.0$ with $R e=7760$, and subcritical for $a=1.5$ at $R e=$ 7220 , and for $a=2.0$ with $R e=5120$.

For the current numerical experiment, a $47 \times 47$ mildly clustered finite element mesh is used to spatially discretize the incompressible Navier-Stokes equations. The flow solver is the finite element code FIDAP. Nine-node quadrilateral elements with biquadratic interpolation functions for velocity components are used. The bilinear pressure interpolation functions are projected onto the four Gauss points inside each element. In order to reduce the number of nodal unknowns, a penalty approach to remove the pressure is used. The elements are 5 times thinner at the side walls and the bottom and 7 times thinner at the moving lid boundary than at the cavity center. After the spatial discretization, and the use of the weighted residual Galerkin method and the penalty formulation for the pressure, we obtain

$$
\mathbf{M} \frac{d \mathbf{U}}{d t}+\mathbf{K}(\mathbf{U}) \mathbf{U}=\mathbf{F} .
$$

Here $\mathbf{U}$ is the global vector of system unknowns of size $2 * N$ where $N$ is the total number of non-boundary nodes. $\mathbf{M}$ is a block diagonal mass matrix. The nonlinear matrix $\mathbf{K}$ represents contributions from the convective and diffusive terms. $\mathbf{F}$ is a generalized force vector which includes contribution from body forces.

The dynamics of two implicit predictor-corrector time integrators are studied. The first is a first-order implicit Euler

$$
\mathbf{M} \frac{\mathbf{U}^{n+1}-\mathbf{U}^{p}}{h}+\mathbf{K}\left(\mathbf{U}^{n+1}\right) \mathbf{U}^{n+1}=\mathbf{F}^{n+1},
$$

with the explicit Euler scheme as predictor

$$
\mathbf{M} \frac{\mathbf{U}^{p}-\mathbf{U}^{n}}{h}+\mathbf{K}\left(\mathbf{U}^{n}\right) \mathbf{U}^{n}=\mathbf{F}^{n} .
$$


The second is the Trapezoidal rule

$$
\mathbf{M} \frac{\mathbf{U}^{n+1}-\mathbf{U}^{p}}{h_{n}}+\frac{1}{2}\left[\mathbf{K}\left(\mathbf{U}^{n+1}\right) \mathbf{U}^{n+1}+\mathbf{K}\left(\mathbf{U}^{p}\right) \mathbf{U}^{p}\right]=\frac{1}{2}\left[\mathbf{F}^{n+1}+\mathbf{F}^{p}\right],
$$

with the Adams formula as predictor

$$
\mathbf{U}^{p}=\mathbf{U}^{n}+\frac{h_{n}}{2}\left[\left(2+\frac{h_{n}}{h_{n-1}}\right) \dot{\mathbf{U}}^{n}-\frac{h_{n}}{h_{n-1}} \dot{\mathbf{U}}^{n-1}\right],
$$

where $h$ is a constant time step, $h_{n}$ is a variable time step and $\dot{\mathbf{U}}^{n}$ is an acceleration vector, approximated by

$$
\dot{\mathbf{U}}^{n}=\frac{2}{h_{n-1}}\left(\mathbf{U}^{n}-\mathbf{U}^{n-1}\right)-\dot{\mathbf{U}}^{n-1} .
$$

The local time truncation error $\Delta \mathbf{U}^{n+1}$ is computed as follows:

$$
\Delta \mathbf{U}^{n+1}=\frac{\mathbf{U}^{n+1}-\left(\mathbf{U}^{n+1}\right)^{p}}{3\left(1+\frac{h_{n-1}}{h_{n}}\right)}+O\left(h_{n}^{4}\right) .
$$

After the norm $\left\|\Delta \mathbf{U}^{n+1}\right\|$ is evaluated, the next time step is computed according to the formula

$$
h_{n+1}=h_{n}\left(\frac{\epsilon}{\left\|\Delta \mathbf{U}^{n+1}\right\|}\right)^{1 / 3}
$$

where $\epsilon$ is a truncation error tolerance. We also set an upper limit, $h_{\max }$, that restricts growth of the time step.

We first study the dynamics of these time integrators using a fixed time step ( $h_{k}=$ constant). Standard Newton-Raphson and quasi-Newton iterative methods are used to solve the nonlinear algebraic equations for this system of ODEs with the predicted solution as an initial guess. With a fixed time step of $h=0.001$, both time integrators produce a periodic solution. However, as the time step is increased up to $h=0.01$, the first time integrator is attracted to the steady state solution. This phenomenon of spurious stabilization of an unstable steady state is very typical for implicit LMM schemes. This spurious steady state remains stable as $h$ increases further.

The dynamics of the second integrator is different. The solution remains time periodic up to $h=0.75$. For $h$ between 0.75 and 0.8 , the solution appears quasiperiodic, indicating the occurrence of the secondary Hopf bifurcation. With $h$ increased to 1.0 , the quasiperiodic oscillations become increasingly disturbed until the solution appears very irregular for $h>1.0$, which is indicative of numerically induced chaos. With further increases in $h$, more complex bifurcations occur with the computed solution becoming regular again at $h=1.3$ 


\section{2-D Lid Driven Cavity (LDC) (Joint work with M. Poliashenko) (Different Time Integrators Exhibit Distinct Spurious Bifurcations)}

Numerical Methods: (Finite Element Code - FIDAP)

Space: Weighted residual Galerkin method + penalty approach to remove $p$

Time: Implicit Euler \& trapezoidal rule

Newton-Raphson \& Quasi-Newton to solve the nonlinear algebraic Eqns.

Explicit Euler \& Adams formula as initial guesses

BCs: $v(y=a)=1$

Rest of BCs are zero

Grid: $47 \times 47$

$\underline{\underline{R e}}=5500$

\section{Summary:}

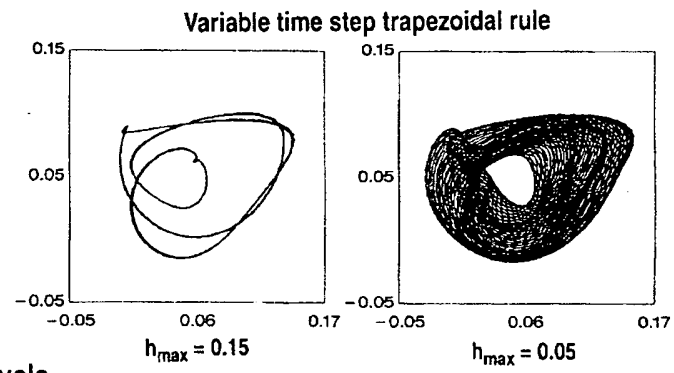

Implicit Euler: $h=0.005$ - limit cycle $h=0.01$ - steady state (stabilizing unstable $S S$ )

Trapezoidal: $h=0.75$ - limit cycle

$h=0.8$ - quasi periodic (secondary bifurcation) $h>0.1$ - chaotic behavior variable time step - different spurious behavior (Poliashenko \& Aidun, 1995)

Figure 6.1. Two different predictor-corrector implicit methods exhibit distinct spurious bifurcations. Phase portraits using a variable time step predictor-corrector implicit method (Trapezoidal rule as corrector and third-order Adams-Bashforth as predictor). (Left figure: $h_{\max }=0.15$. Right figure: $h_{\max }=0.05$ ). 
and then returning to chaotic behavior. With fully developed chaotic behavior and the solution being non-smooth, more and more singular eruptions occur. This makes it difficult for the Newton-Raphson procedure to converge. At some point around $h=1.5$, the Newton-Raphson method fails to converge, implying that there is a nonlinear instability of the chosen time integrator at this time step.

Similar behavior of the numerical solution is observed if the quasi-Newton procedure is applied instead of the standard Newton-Raphson method in solving the underlying nonlinear algebraic system. However, in this case quasiperiodic solutions tend to become irregular with smaller time steps and the transition to chaos occurs earlier.

In Poliashenko \& Aidun (1995) the variable time step control version of the Adams-Bashforth/Trapezoidal predictor-corrector scheme with truncation error tolerance of 0.005 and maximum time step of 0.15 for variable time step control and the quasi-Newton nonlinear solver are used. They found that after $R e>$ 6200 , a weak modulation of the oscillation envelope occurs and the solution becomes quasiperiodic with the indication of a secondary Hopf bifurcation. As $R e$ approaches 6500 , they observed a strong resonance between the two independent frequencies which transformed the solution from quasiperiodic to strictly periodic. This phenomenon is known in dynamical systems as "phaselock" on the torus. This resulted in the birth of limit cycles. This limit cycle is observed in a fairly wide range of $R e$ up to 6700 . At $R e=6700$, the numerical solution exhibits more complex bifurcations and transitions to weakly turbulent, or chaotic motion.

As we decrease $h_{\max }<0.12$, the limit cycle shown in Fig. 6.1 is replaced by a stable 2-D torus which remains qualitatively unchanged as $h$ is further decreased and appears to be close to the "true" solution of the ODEs. The latter example demonstrates that a variable time step integrator with local error control, although more reliable, does not guarantee no spurious numerics.

\subsection{Chaotic Transients Near the Onset of Turbulence in Direct Numerical Simulations of Channel Flow (Keefe 1988, Yee \& Sweby 1997)}

In addition to the inherent chaotic and chaotic transient behavior in some physical systems, numerics can independently introduce and suppress chaos as well as chaotic transients. Loosely speaking, a chaotic transient behaves like a chaotic solution (Grebogi et al. 1983). A chaotic transient can occur in a continuum or a discrete dynamical system. One of the major characteristics of a numerically induced chaotic transient is that if one does not integrate the discretized equations long enough, the numerical solution has all the character- 
istics of a chaotic solution. The required number of integration steps might be far beyond those found in standard CFD simulation practice before the numerical solution can get out of the chaotic transient mode. Furthermore, standard numerical methods, depending on the initial data, usually experience drastic reductions in step size and convergence rate near a bifurcation point of the continuum in addition to the bifurcation points due solely to the discretized parameters. See Yee \& Sweby $(1992,1995,1996,1997)$ for a discussion. Consequently, a possible numerically induced chaotic transient is especially worrisome in direct numerical simulations of the transition from laminar to turbulent flows. Except for special situations, it is extremely difficult to bracket closely the physical transition point by mere DNS of the Navier-Stokes equations. Even away from the transition point, this type of numerical simulation is already very CPU intensive and the convergence rate is usually rather slow. Due to limited computer resources, the numerical simulation can result in chaotic transients indistinguishable from sustained turbulence, yielding a spurious picture of the flow for a given Reynolds number. Consequently, it casts some doubt on the reliability of numerically predicted transition points and chaotic flows. It also influences the true connection between chaos and turbulence. See also Moore et al. (1990).

Assuming a known physical bifurcation or transition point, Fig. 6.2 illustrates the schematic of four possible spurious bifurcations due to constant time steps and constant grid spacings. This section and the next (Section 6.4) illustrate the occurrence of these scenarios. Section 6.4 discusses the stability of the steady state (as a function of the Reynolds number) of a 2-D backward facing step problem using direct simulations. The present section is the computation by Laurence Keefe performed in the late 1980s. In 1996 we made use of the knowledge from continuum and discrete dynamical systems theory to interpret his result. We identified some of the aforementioned numerical uncertainties in his computations. The result is reported in Yee \& Sweby 1997.

The physical problem that Keefe considered is depicted in Fig. 6.3, where a flow is confined between planes at $y= \pm 1$ and is driven in the $x$-direction by a mean pressure gradient $d p / d x$. The flow is characterized by a Reynolds number $R e=U_{\infty} L / \nu$, where $U_{\infty}$ is the mean centerline velocity, $L$ is the channel half-height, and $\nu$ is the kinematic viscosity. Within the channel the flow satisfies the incompressible Navier-Stokes equations and no-slip boundary conditions are applied at the walls. In the particular calculations shown here these equations have been manipulated into velocity-vorticity form, where one integrates equations for the wall-normal velocity $v$ and normal vorticity $\eta$, and recovers the other two velocity components from the incompressibility condition and the definition of $\eta$. 


\section{Schematic of Possible Spurious Bifurcation (Assume a certain physical transition; same IC \& BC)}

1. Differenl temporal discrel. (same spatlal discret)

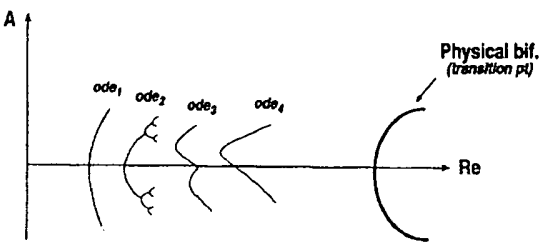

2. Dlfferent di (same spatial \& temporal discret)

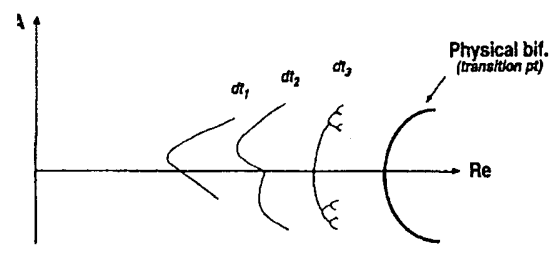

3. Different dx (same spatial \& temporal discret.)

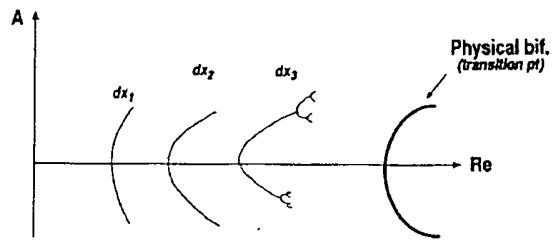
4. Differeni spatial discret. (same temporal discret)
(Mred of a da)

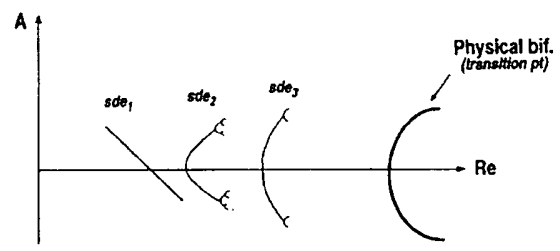

Figure 6.2. Schematic of possible spurious bifurcation for constant time steps and grid spacings. (1) Different temporal discretizations ode od $_{1}$ ode $_{2}$, ode od $_{3}$ and ode od $_{4}$ (same spatial discretization and the same constant $d t$ and $d x$ ). (2) Different constant time steps $d t_{1}, d t_{2}, d t_{3}$ and $d t_{4}$ (same temporal and spatial discretizations, and the same constant $d x$ ). (3) Different constant grid spacings $d x_{1}, d x_{2}, d x_{3}$ and $d x_{4}$ (same spatial and temporal discretizations, and the same constant dt). (4) Different spatial discretizations $s_{1} e_{1}, s_{d} e_{2}$ and sde $_{3}$ (same temporal discretization and the same constant $d t$ ). 


$$
\begin{gathered}
\frac{\partial}{\partial t} \Delta^{2} v=h_{v}+\frac{1}{R e} \Delta^{4} v, \\
\frac{\partial}{\partial t} \eta=h_{g}+\frac{1}{R e} \Delta^{2} \eta, \\
f+\frac{\partial v}{\partial y}=0,
\end{gathered}
$$

where

$$
\begin{gathered}
f=\frac{\partial u}{\partial x}+\frac{\partial w}{\partial z}, \quad \eta=\frac{\partial u}{\partial z}-\frac{\partial w}{\partial x} \\
h_{v}=-\frac{\partial}{\partial y}\left(\frac{\partial H_{1}}{\partial x}+\frac{\partial H_{3}}{\partial z}\right)+\left(\frac{\partial^{2}}{\partial x^{2}}+\frac{\partial^{2}}{\partial z^{2}}\right) H_{2}, \\
h_{g}=\frac{\partial H_{1}}{\partial z}-\frac{\partial H_{3}}{\partial x} .
\end{gathered}
$$

Here the $H_{i}$ contain the nonlinear terms in the primitive form of the NavierStokes equations and the mean pressure gradient.

The velocity increases extremely rapidly normal to the wall, and turbulent channel flows are essentially homogeneous in planes parallel to the wall. The first requires a concentration of grid points near the wall, and the second suggests use of a doubly periodic domain in planes parallel to the wall. A spectral representation of the velocity field $(u, v, w)$

$$
\vec{u}=\sum_{l} \sum_{m} \sum_{n} \vec{A}_{l m n}(t) T_{l}(y) e^{i m \alpha x+i n \beta z}
$$

where the $T_{l}(y)$ are Chebyshev polynomials used for the spatial discretization. The numerical problem then becomes dependent on $\alpha$ and $\beta$ in addition to Re. For the time discretization, mixed explicit-implicit methods are used. The nonlinear terms in the equations are advanced using second-order AdamsBashforth or a low storage, third-order Runge-Kutta scheme (Spalart et al. 1991), while the viscous terms are advanced by Crank-Nicholson.

One of the central problems in studies of wall bounded shear flows is the determination of when a steady laminar flow becomes unstable and transitions to turbulence. In dynamical systems terms, the Navier-Stokes equations always have a fixed point solution for low enough Reynolds numbers, but for each flow geometry the Reynolds number at which this fixed point bifurcates needs to be determined. In channel flow the fixed point solution (a parabolic velocity profile across the channel, $\left.u(y)=\left(1-y^{2}\right)\right)$ becomes linearly unstable at $R e=5,772$ (Orszag 1971). However, since turbulence appears in experiments at much 
lower Reynolds numbers, it was conjectured that this bifurcation must be subcritical. Subsequent numerical solution of the nonlinear stability equations (Herbert 1976, Ehrenstein \& Koch 1991) demonstrated this to be true, showing that limit cycle solutions with amplitude $\epsilon$ branch back to lower Reynolds numbers before subsequently passing through a turning point and curving back toward higher Reynolds numbers. Thus for Reynolds numbers just above the turning point the flow equations have at least four solutions: the fixed point; two unstable limit cycles; and a chaotic solution (experimentally observed turbulence). Determining the location of the turning point in $(\alpha, \beta, \epsilon, R e)$ space is known as the minimum-critical-Reynolds-number problem, and its solution is by no means complete.

One way to investigate the turning point problem is to perform DNS of channel flow for conditions believed to be near this critical condition. Beginning with a known turbulent initial condition from higher Reynolds number, one integrates in time at the target Reynolds number to determine whether the flow decays back to the fixed point or sustains itself as turbulence. Although this may not be the most efficient way to bracket the turning point, it has the advantage that the peculiar dynamics of the flow near the turning point, whether in decay or sustained turbulence, are observable. This yields information about the path along which flows become turbulent at these low Reynolds numbers.

Unfortunately the flow dynamics are very peculiar near the turning point, and extremely long chaotic transients are observed in the computations that make a fine determination of that point all but impossible by this method. This can be seen in Fig. 6.3, where a time history of the turbulent energy in a channel flow (energy above that in the laminar flow) is plotted for a Reynolds number of 2,191. To understand the time scale of the phenomenon some experimental facts need to be recalled. In typical experimental investigations of channel flow, the infinite transverse and streamwise extent of the ideal flow are approximated by studying flow in high aspect ratio (10-40) rectangular ducts that typically are 50-100 duct heights long. If times are non-dimensionalized by the centerline mean velocity $U_{\infty}$ and the duct half height $L$, then statistics on turbulence are gathered by averaging hot-wire data over intervals $\Delta t U_{\infty} / L \sim 200$. In the simulations and figure the time scale is based on the friction velocity $u_{\tau}$ and $L$, where typically 15-20 $u_{\tau} \sim U_{\infty}$. Thus averaging over intervals $\Delta t u_{\tau} / L \sim 10$ should and does yield stable flow statistics that compare well with experiments. The near-wall velocity profile, cross- channel turbulence intensities, and Reynolds and shear stress distribution for the $\Delta t u_{\tau} / L \sim 10$ interval near the end of the transient, delineated by the arrows in Fig. 6.3, indicate the good comparison. In each case they correspond well to available experimental data. Yet look at the time scale of the transient; it spans $\Delta t u_{\tau} / L \sim 300$, thirty times longer than the time needed to obtain stable statistics that would convince most experimentalists 


\section{Chaotic Transient Near the Onset of Turbulence} (Keefe 1996)

Re $=2191,32 \times 33 \times 32$ grid, mixed expllcit \& implicit spectral method 163,840 steps, transient calculatlon length of $409.6,40$ hrs on Cray XMP

$$
\begin{aligned}
R e= & U_{\infty} L / v \\
& U_{\infty}-\text { mean centerline velocity } \\
& L-\text { channel half height } \\
& v-\text { kinematic viscosity }
\end{aligned}
$$

Geometry of Polseullie Flow

Aspect Ratio of Duct: -3

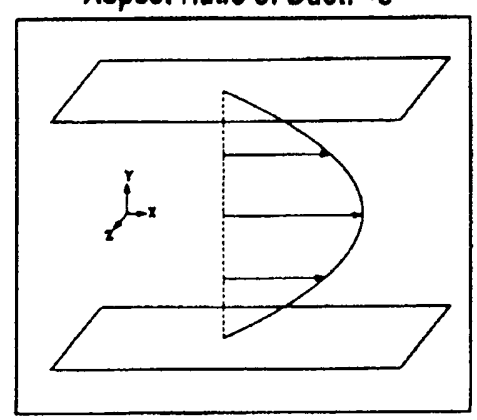

Keefe et al. (1992):
Time History of the Turbulent Energy

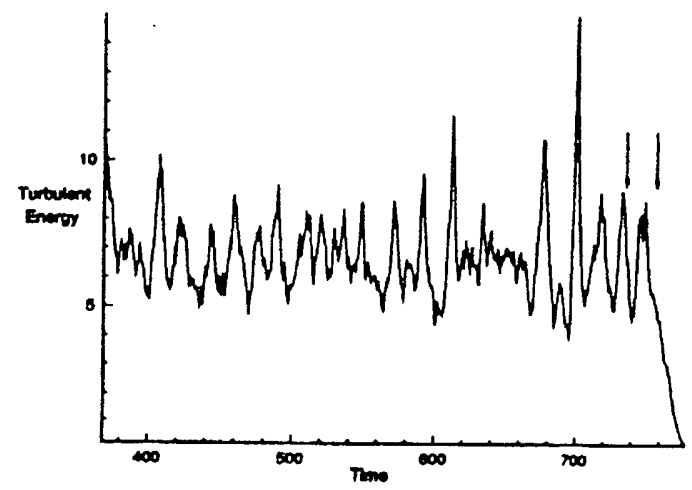

Performed the "dimension \& Lyapunov exponent" study at midpoint of later computation ( $R_{\theta}=3200,429,680$ steps)

Figure 6.3. 3-D channel flow computation by Keefe, 1988. 
that they are viewing a fully developed turbulent channel flow. This is further complicated by the wide variation of the transient length, depending upon both the grid resolution (number of modes in the spectral representation) and the linearly stable time step of the integration. In fact, for fixed $(\alpha, \beta, R e)$ it is possible to obtain sustained turbulence for one time step, but see it rapidly decay to laminar flow for another, lower value of the step.

Extended chaotic transients near bifurcation points are not an unknown phenomenon; the "meta-chaos" of the Lorenz system is but one of many known examples. However, the practicalities of numerical computation in fluid dynamics usually interfere with one's ability to discern whether transient, or sustained turbulence, is being calculated. The computations required to obtain the transient plot in Fig. 6.3 needed 40 hours of single processor time on a Cray XMP, some ten years ago. Such a small amount of expended time was only possible because the spatial resolution of the calculation was relatively coarse $(32 \times 33$ $x 32$ ), in keeping with the large scales of the phenomena expected at these flow conditions. Higher resolution calculations (192 x $129 \times 160)$ (Kim et al. 1987) at greater Reynolds numbers typically have taken hundreds of hours $(\sim 250)$ to barely obtain the $\Delta t u_{\tau} / L=10$ averaging interval that is so inadequate for detecting transients. Because such calculations are so time consuming, one typically chooses an integration time step that is a substantial fraction of the linear stability limit of the algorithm, so as to maximize the calculated "flow time" for expended CPU time. However, it is clear from these transient results that this practice has some dangers when close to critical points of the underlying continuous dynamical system. Thus it appears that just as pseudo-time integration to obtain steady solutions can result in spurious results, genuine time integration can result in chaotic transients indistinguishable from sustained turbulence, also yielding a spurious picture of the flow for a given Reynolds number.

\subsection{Temporal \& Spatial Refinement Studies of 2-D Incompressible Flow over a Backward-Facing Step}

The 2-D incompressible flow over a backward-facing step has been addressed by many authors using a wide variety of numerical methods. Figure 6.4 shows the flow geometry. Fluid with constant density $\rho$ and viscosity $\mu$ enters the upstream channel of height $h$ with a prescribed velocity profile (usually parabolic). After traveling a distance $l$, the fluid passes over a backward-facing step of height $s$ and enters the downstream channel of height $H=h+s$. After traveling a distance $L$ downstream of the step, the fluid exits the region of interest. For Reynolds numbers considered here, the flow separates at the corner and forms a recirculating region behind the step. Additional recirculating regions form on 


\section{Backward Facing Step \\ (2-D Incompressible Flow Simulations)}

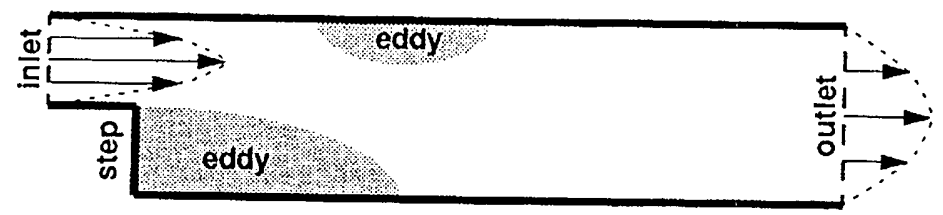

\section{Early 90's Controversy: Transition point Reynolds \#}

- Reports of sustained unsteady flow for Reynolds \# in the range of $(250,2500)$

- Formulations

vortex method, unsteady Eqns. in stream function form, unsteady Eqns. \& the associated linear-stability problem, unsteady Egns. in primitive variable form

- Numerical Methods

All of the existing schemes in the literature

\section{Gresho et al. (1993): Provided an answer to the above controversy} (the steady solution at $R e=800$ is stable)

- Kaiktsis et al. (1991) - transition to turbulent flow has occurred at $\mathrm{Re}=800$

- Torczynski (1993) - the result of Kaiktsis et al. (1991) is an artifact of inadequate spatial resolution

- Torczynski's conclusion was confirmed by a subsequent study of Kaiktsis et al. (1996) \& Fortin et al. (1996)

Figure 6.4. Schematic of the backward facing step problem and background. 
the upper and subsequently the lower walls of the downstream channel as the Reynolds number is increased.

Results of sustained unsteady flow from various numerical simulations have been reported for Reynolds numbers ( $R e$ ) ranging from 250 up to 2500. The formulations included the vortex method, unsteady equations in stream function form, steady equations and the associated linear-stability problem, and the unsteady equations in primitive variable form. The numerical methods used cover almost all of the existing schemes in the literature. The majority of the numerical results are summarized in Gresho et al. (1993). The work of Gresho et al. was an answer to a controversy concerning the stability of the stationary solution at $R e=800$. It was concluded by Kaiktsis et al. (1991) that transition to turbulent flow has occurred at $R e=800$. Kaiktsis et al. examined the long-time temporal behavior of the flow and found that the flow is steady at $R e=500$, time-periodic at $R e=700$, and chaotic at $R e=800$. Gresho et al. did a detailed grid refinement study using four different numerical methods and concluded that the backward-facing step at $R e=800$ is a stable steady flow.

In addition to the study of Gresho et al., an extensive grid refinement study of this flow using a spectral element method was conducted in Torczynski (1993). The simulated geometry and the numerical method corresponds to that of Kaiktsis et al. (1991). Flow was examined at Reynolds numbers of 500 and 800. His systematic grid refinement study was performed by varying both the element size and the order of the polynomial representation within the elements. For both Reynolds number values with the transient computations stopped at $t=800$, it was observed that low-resolution grid cases exhibit chaotic-like temporal behavior whereas high-resolution grid cases evolve toward asymptotically steady flow by a monotonic decay of the transient. The resolution required to obtain asymptotically steady behavior is seen to increase with Reynolds number. These results suggest that the reported transition to sustained chaotic flow (Kaiktsis et al., 1991) at Reynolds numbers around 700 is an artifact of inadequate spatial resolution. Torczynski's conclusion was further confirmed by a subsequent study of Kaiktsis et al. (1996) and Fortin et al. (1996). Fortin et al. employed tools from dynamical systems theory to search for the Hopf bifurcation point (transition point). They showed that the flow remains steady at least up to $R e=1600$.

Grid Refinement Study of Torczynski (1993): In Torczynski (1993), the $R e=\rho \bar{u} 2 h / \mu$ is based on upstream conditions. The variable $\bar{u}$ is the spatial average of the horizontal velocity $u$ over $h$. The geometry is specified to match that of Kaiktsis et al. (1991). The upstream channel height $h$ and step height $s$ have values of $h=1$ and $s=0.94231$, yielding a downstream channel height of $H=1.94231$. The corner of the step is at $(x, y)=(1,0)$. The channel 
extends a distance $L=1$ upstream from the step and a distance $L=34$ downstream from the step to preclude undue influence of the finite channel length on the flow at $R e=800$. The following conditions are applied on the boundaries of the computational domain: $u=v=0$ on the upper and lower channel walls, $-p+\mu \partial u / \partial n=0$ and $\partial v / \partial n=0$ on the outflow boundary, and $u=[\tanh (t / 16)] u_{B}(y)+[1-\tanh (t / 16)] u_{P}(y)$ and $v=0$ on the inflow boundary and the step surface. Here, $u_{B}(y)=\max [0,3 y(1-y)]$ is the correct boundary condition for flow over a backward-facing step and $u_{P}(y)=3(1-y)(s+y) /(1+s)^{3}$ is the Poiseuille flow observed infinitely far downstream whenever steady flow is asymptotically obtained. The initial velocity field is set equal to $u=u_{P}(y)$ and $v=0$ throughout the domain. Here $v$ is the vertical velocity and $p$ is the pressure. Thus, the above combination of boundary and initial conditions initially allows flow through the step surface so that the simulations can be initialized using an exact divergence-free solution of the Navier-Stokes equations. Furthermore, since the inflow boundary condition is varied smoothly in time from Poiseuille flow to flow over a backward-facing step, the flow experiences an order-unity transient that is probably strong enough to excite sustained unsteady behavior, if that is the appropriate asymptotic state for the numerical solution.

The simulations were performed using the commercial code NEKTON v2.8, which employs a time-accurate spectral-element method with the Uzawa formulation (NEKTON, 1991). Let $D$ be the dimensionality. Each element has $N^{D}$ velocity nodes located at Gauss-Lobatto Legendre collocation points, some of which are on the element boundaries, and $(N-2)^{D}$ pressure nodes located at Gauss Legendre collocation points, all of which are internal. Within each element, the velocity components and the pressure are represented by sums of $D$-dimensional products of Lagrangian-interpolant polynomials based on nodal values. This representation results in continuous velocity components but discontinuous pressure at element boundaries. Henceforth, the quantity $N$ is referred to as the element order, even though the order of the polynomials used to represent the velocity is $N-1$. NEKTON employs mixed explicit and implicit temporal discretizations. To avoid solving a nonlinear nonsymmetric system of equations at each time step, the convective term is advanced explicitly in time using a third-order Adams-Bashforth scheme. All other terms are treated implicitly (implicit Euler for the pressure and for the viscous terms).

Three spectral-element grids of differing resolution, denoted $L$ (low), $M$ (medium), and $H$ (high), are employed. Figure 6.6 shows the computational domain and the grid distribution of the three spectral element grids in which the distribution of nodes within each spectral element is not shown. The $L$ grid with $N=9$ is identical to the grid of Kaiktsis et al. (1991). Four general classes of behavior are observed for the numerical solutions. First, "steady monotonic" 
denotes evolution of the numerical solution toward an asymptotically steady state. Second, "steady oscillatory" denotes evolution toward an asymptotically steady state with a decaying oscillation superimposed on the monotonic decay. Third, "unsteady chaotic" denotes irregular transient behavior of the numerical solution that shows no indication of evolving toward steady behavior. Fourth, "diverge" denotes a numerical solution terminated by a floating-point exception. In Fig. 6.6, the first character denotes the grid resolution $L, M$ or $H$, the first digit indicates the Reynolds number 500 or 800 and the last two digits indicate the order of the spectral element being used. For example, $L 807$ means $R e=800$ using the $L$ grid with $N=7$.

The extensive grid refinement study of Torczynski resulted in grid-independent steady-state numerical solutions for both $R e=500$ and $R e=800$. As the grid resolution is reduced below the level required to obtain grid independent solutions, chaotic-like temporal behavior occurred. The degree of grid resolution required to obtain a grid-independent solution was observed to increase as the Reynolds number is increased. Figure 6.5 shows the streamlines for for $H 809$ (steady solution) and $L 811$ (spurious time-periodic solution) and the corresponding grids with the distribution of the nodes of the spectral elements shown.

\section{Temporal Refinement Studies Using Knowledge from Dynamical Systems}

Theory: All of Torczynski's numerical solutions integrate to $t=800$. With the knowledge of possible nonlinear behavior of numerical schemes such as long time transients before a steady state is reached, numerically induced chaotic transients, numerically induced or suppressed chaos, existence of spurious steady states and asymptotes, and the intimate relationship among initial data, time step and grid spacing observed in discrete dynamical systems theory, Yee et al. (1997) examined the Torczynski cases in more detail.

In the Yee et al. (1997) study, in addition to grid refinement, temporal refinements are made on all of the under-resolved grid cases to determine if these cases sustain the same temporal behavior at a much later time or evolve into a different type of spurious behavior. At $t=800$, cases $L 506, L 507, L 508, L 509, L 811$, $M 807$ and $M 808$ either exhibit "unsteady chaotic" or "steady oscillatory" behavior. We integrate these cases to $t=2000$ to determine if a change in solution behavior occurs. From the phenomena observed in Keefe's 3-D channel flow computation and others, $t=2000$ might not be long enough for a long time transient or long chaotic transient to die out. There is also the potential of evolving into a different type of spurious or divergent behavior at a much later time. However, for this study it appears that $t=2000$ is sufficient. For $R e=500$, we also recomputed some of these cases with a sequence of $\Delta t$ that bracketed the benchmark study of Torczynski. The $\Delta t$ values are 


\section{Backward Facing Step Simulations
(By a spectral element computer code NEKTON)}

3 Grids (L, M \& H)

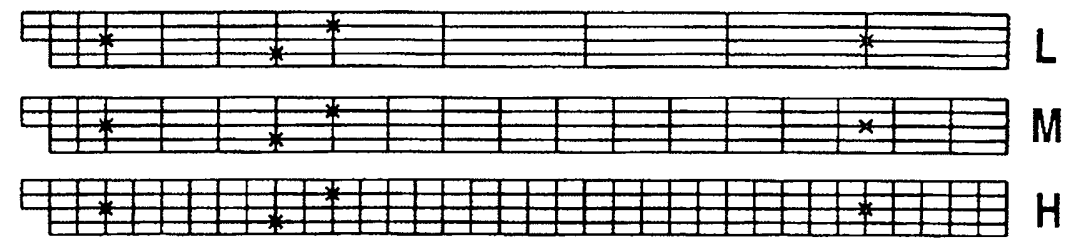

\section{Streamlines \& Grids}

$\mathrm{Re}=800, \mathrm{t}=2000$ (vertical expanded 4 times)

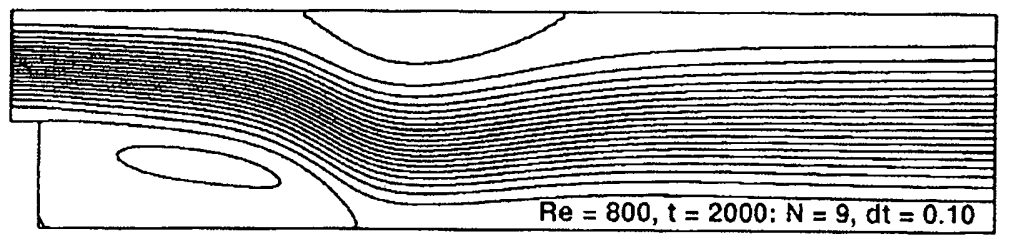

Correct

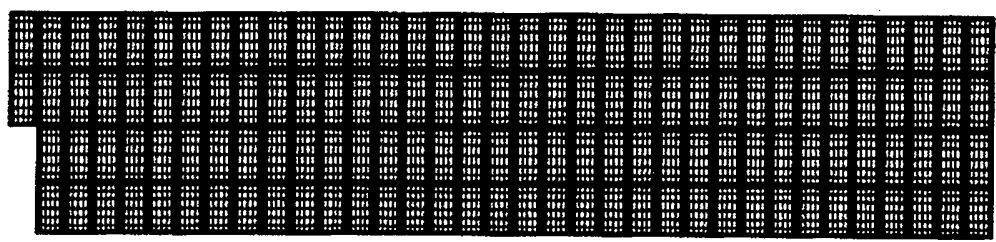

$H$ grid
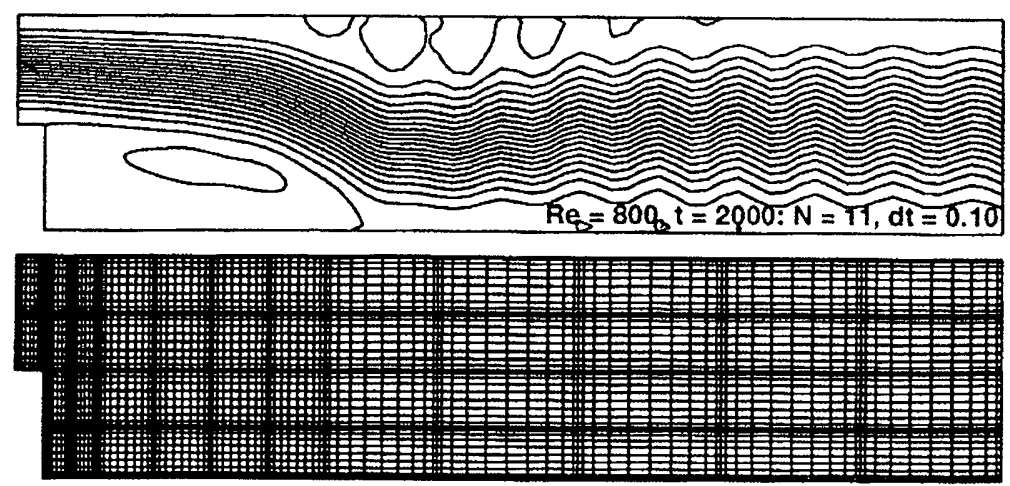

L grid

Figure 6.5. Three different grids, and streamlines of H809 and L811 and their corresponding grids by the spectral element method. 
$0.02,0.05,0.10,0.125,0.2,0.3,0.4$, and 0.5 for $R e=500$. The CFL number for all of these cases is above 1 for $\Delta t>0.10$. The reason for the investigation of $\Delta t=0.3,0.4$ and 0.5 is to find out, after the transients have died out, if the solution converges to the correct steady state for $\Delta t$ that are a few times larger than 0.10 .

For $R e=800$, we integrate $L 811$ and $M 808$ with $\Delta t=0.10$ and $M 807$ with $\Delta t=0.02,0.05$ and 0.10 to $t=2000$. Aside from integrating to $t=2000$, five different initial data were examined for cases $M 807, M 809$ and $M 811$ for $\Delta t=0.10$ to determine the influence of the initial data and the grid resolution on the final numerical solution. The five initial data are:

(a) Uniform: $u, v=0$

(b) Shear layer: $u=u_{B}(y)=\max [0,3 y(1-y)], v=0$

(c) Solution from solving the steady Stokes equation (with no convection terms)

(d) Torczynski (1993): $u=u_{P}(y)=3(1-y)(s+y) /(1+s)^{3}, v=0$

(e) Channel flow both upstream \& downstream of step: Same as (d) except the boundary conditions

The boundary conditions for (a), (b), (c) and (e) were parabolic inflow and noslip at walls, whereas the boundary conditions for (d) were those of Torczynski (1993): $u=[\tanh (t / 16)] u_{B}(y)+[1-\tanh (t / 16)] u_{P}(y)$ and $v=0$. The CPU time required to run the above cases ranged from less than a day to several days on a Sparc Center 2000 using one processor.

The chaotic-like behavior evolves into a time-periodic solution beyond $t=$ 800 for $L 506$ and $L 507$, whereas the chaotic-like behavior evolves into a timeperiodic solution beyond $t=800$ for $L 811$ and a divergent solution for $M 807$. The "steady oscillatory" case $L 508$ slowly evolves to the correct steady state with an amplitude of oscillation of $10^{-5}$. The oscillation is not detectable within the plotting accuracy. The "steady oscillatory" time evolution of M808 is similar to that of $L 508$. The numerical solutions with "steady oscillatory" and "steady monotonic" behavior at early stages of the time integration are almost identical at later stages of the time integration. They all converge to the correct steady state. The initial data study at $R e=800$ with $\Delta t=0.10$ is summarized in Table 5.5 of Yee et al. (1997). It illustrates the intimate relationship between initial data and grid resolution.

Figure 6.6 shows the vertical velocity time histories at $(x, y)=(30,0)$ advanced to a time of $t=2000$ for $M 807$ with $\Delta t=0.02,0.05$ and 0.10 , and $L 811$ for $\Delta t=0.10$. Case $M 807$ diverges at $t=1909.2$ for $\Delta t=0.02$, at $t=972.4$ for $\Delta t=0.05$, and at $t=827.77$ for $\Delta t=0.10$. The time histories for these three time steps appear to show chaotic-like behavior if one stops the computations at $t=800$. The bottom plot of Fig. 6.6 shows the 


\section{Vertical Velocity Time Histories at $(30,0)$ $\mathrm{Re}=800, M$ \& L Grids}
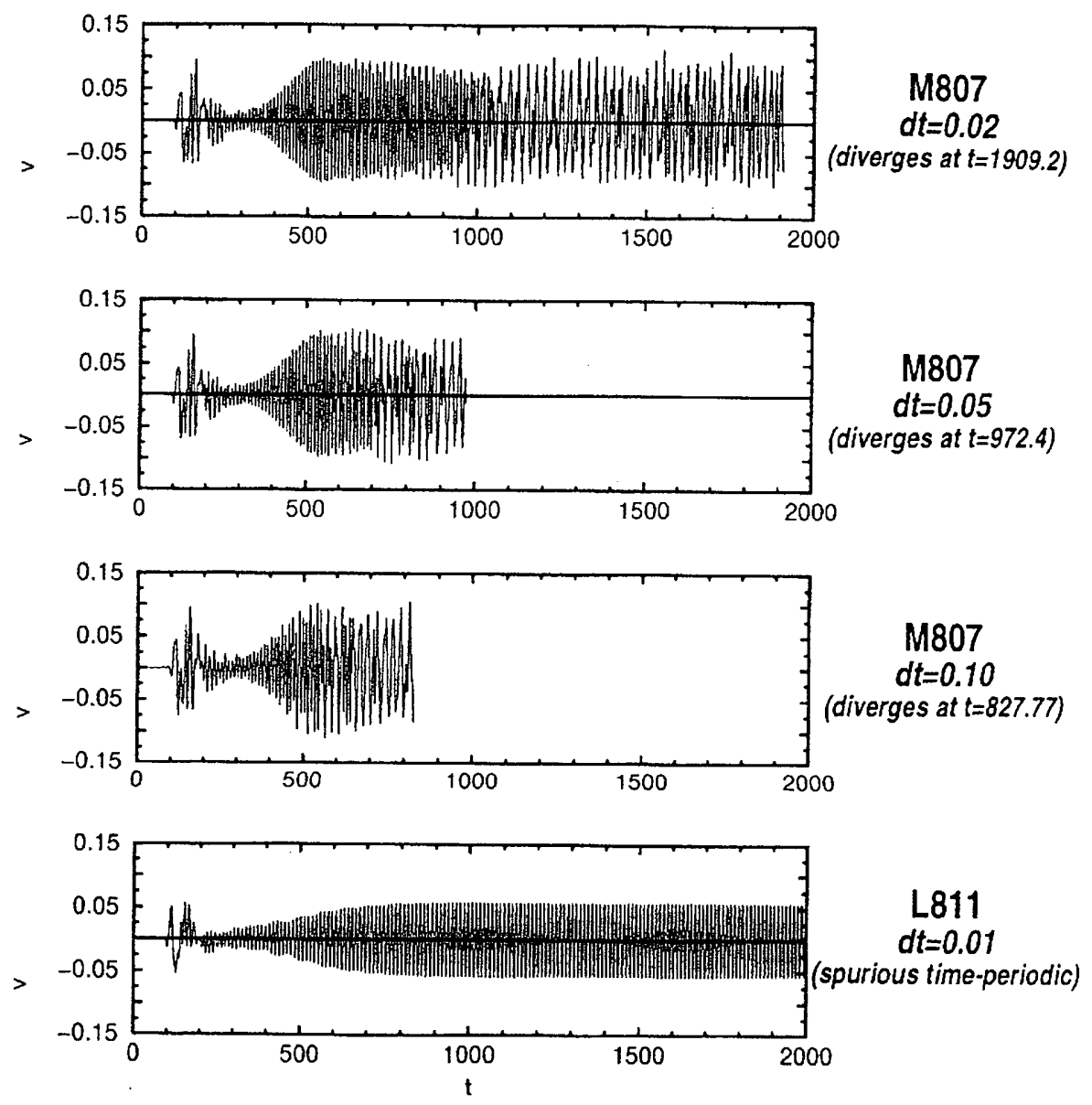

Figure 6.6. Vertical velocity time histories for the $M 807$ with time steps $0.02,0.05,0.10$, and L811 with time step 0.01 for $t=2000$. 
vertical velocity time histories advanced to a time of $t=2000$ for $L 811$ with $\Delta t=0.10$. It shows the definite time-periodic spurious solution pattern. On the other hand, the time history for this case appears to show an aperiodic-like pattern if one stops the computation at $t=800$. Note that the $L 809$ grid case was used by Kaiktsis et al. (1991) and they concluded that "2-D transition" has already occurred at $R e=800$.

In summary, without the temporal refinement study (longer time integration), the $L 506, L 507, L 811$ and $M 807$ cases can be mistaken to be chaotic-like (or aperiodic-like) flow. Although the time history up to $t=800$ appears chaoticlike, one cannot conclude it is chaotic without longer transient computations. One can conclude that with transient computations that are 2.5 times longer than Torczynski's original computations, what appeared to be aperiodic-like or chaotic-like behavior at earlier times evolved toward either a time-periodic or divergent solution at later times. These temporal behaviors appear to be long time aperiodic-like transients or numerically induced chaotic-like transients. For $R e=800$, five different initial data were examined to determine if the flow exhibits strong dependence on initial data and grid resolution. Results showed that the numerical solutions are sensitive to these five initial data. Note that the results presented pertain to the characteristic of the studied scheme and the direct simulations. However, if one is certain that $R e=800$ is a stable steady flow, a non-time-accurate method such as time-marching to obtaining the steady-state numerical solution would be a more efficient numerical procedure.

Spurious Bifurcation by Different Time Integrators (Henderson \& Yee 1998, unpublished): This is a joint work with Ronald Henderson in 1998. The unpublished work was presented at the 10th International Conference in Finite Element Methods, January 5-8, 1998, Tucson, Arizona, and also has been presented at various invited lectures during the last four years. Our joint work illustrates the situation where solving the nonlinear terms of the Navier-Stokes equations by two different explicit time integrators (same implicit time integrator for the linear terms) results in spurious bifurcation. This spurious bifurcation is shown in Fig. 6.7 as a function of the Reynolds number. These computations use the implicit Euler time integrator for the linear terms. Also the same spatial discretization $L 809$ is used with a fixed time step of $t=0.10$. The two explicit time integrators are the third-order Adams-Bashforth (AB3) and a second-order explicit stiffly stable method (SS2) (Henderson 1999). The AB3 method experiences a spurious bifurcation near $R e=720$, whereas the SS2 method experiences a spurious bifurcation at a larger Reynolds number near $R e=800$. The method and the scaling for this figure can be found in Henderson (1999). Finding the exact location of these spurious bifurcation points requires more complicated computation which is not performed here. In addition, the exact representation of this bifurcation plot is rather complicated to explain, and is not 


\section{Backward Facing Step (Joint work with Henderson \& Torczynski) (Different Time Integrators Exhibit Distinct Spurious Bifurcations)}

\section{Numerical Methods:}

Space: Spectral element (L809 grid)

Time: Implicit Euler (linear term)

AD3 \& SS2 (nonlinear term)

BC \& IC: Same as Torczynski + continuation method Spurious Bifurcations using AD3 \& SS2 (Fixed dt, L809 grid)

\section{Summary: (Explicit time integrators)} - Different time integrators exhibit distinct spurious bifurcations (same grid, same dt)

- As the grid size increases, spurious bifurcations occur at larger Re (same $d t$ )

Adaptive Grid Refinement: (Explicit time integrators) Remains laminar at least up to $R e=1800$

Future Work: (Uncond. stable implicit time integrators)

- Spurious bifurcations occur at larger Re than explicit methods

- Uncond. stable implicit LMMs can stabilize unstable SS as dt increases (same grid)

- Adaptive time \& spatial discretizations

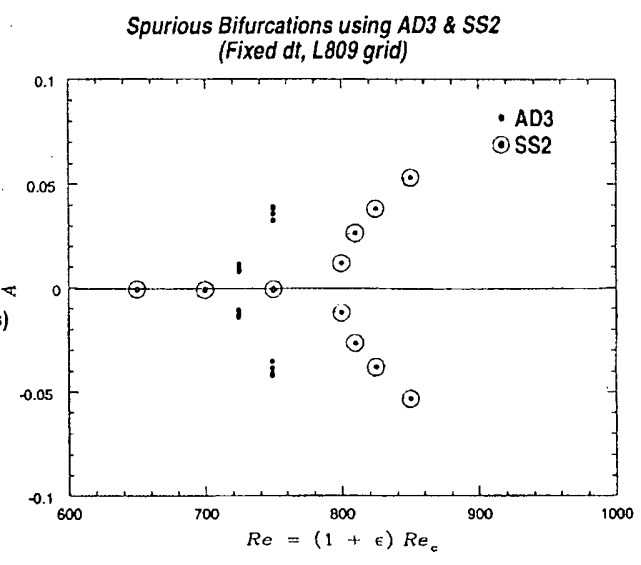

Figure 6.7. Different explicit time integrators (for the nonlinear terms of the Navier-Stokes equations) exhibit distinct spurious bifurcation using the same spatial discretization L809 and a time step of 0.10 . 
important for the current discussion. They are not the main illustration for this study. When an adaptive version of the spectral element method (Henderson, 1999 ) is used, the problem remains laminar up to $R e=1800$. Future work which is indicated on Fig. 6.7 is planned.

Minimization of Spurious Bifurcation by a Suitable Filter (Fischer 2001, unpublished): Recently, Fischer (2001) computed the same $L 811$ spectral element grid using a time integrator based on the operator integration-factor splitting (OIFS) developed by Maday, Patera and Rønquist (1990). This scheme decouples the convective step from the Stokes update, thereby allowing CFL numbers in excess of unity. At the end of each step, Fischer applies a filter to the velocity that effectively scales the $N$ th-order Legendre modes within each element by $(1-\alpha)$, where, typically, $0.05 \leq \alpha \leq 0.30$ (Fischer \& Mullen 2001). Because the filter is applied on each step, its strength is a function of $\Delta t$ as well as $\alpha$. The spurious behavior observed by Kaiktsis et al. (1991) is cured by the filter and a stable steady-state numerical solution is obtained without further grid refinement. Figure 6.8 illustrates the velocity time histories at $(30,0)$ by the filtered and un-filtered spectral element methods with $\Delta t=0.10$.

In summary, Sections 6.3 and 6.4 illustrate all of the possible scenarios of spurious bifurcations indicated on the schematic diagram of Fig. 6.2. The last scenario, discussed briefly at the beginning of Section 6.4, is quite common and is not shown here. See Gresho et al. (1993) and references cited therein for some examples.

\section{Concluding Remarks}

Some building blocks to ensure a higher level of confidence in PAR of numerical simulations have been discussed. The discussion concentrates only on how well numerical schemes can mimic the solution behavior of the underlying PDEs. The possible discrepancy between the chosen model and the real physics and/or experimental data is set aside. These building blocks are based largely on the author's view, background and integrated experience in computational physics, numerical analysis and the dynamics of numerics. They also represent the end result of the various studies with the author's collaborators indicated in the acknowledgment Section. Among many other important building blocks for the PAR of numerical simulations, the author believes the following five building blocks are essential. The first building block is to analyze as much as possible the dynamical behavior of the governing equation. For stability and well-posedness considerations, whenever it is possible, it is also necessary to condition (not pre-condition) the governing PDEs before the application of the appropriate scheme (Yee \& Sjögreen 2001a,b). The second building block is to understand the nonlinear behavior, limits and barriers, and to isolate spurious 

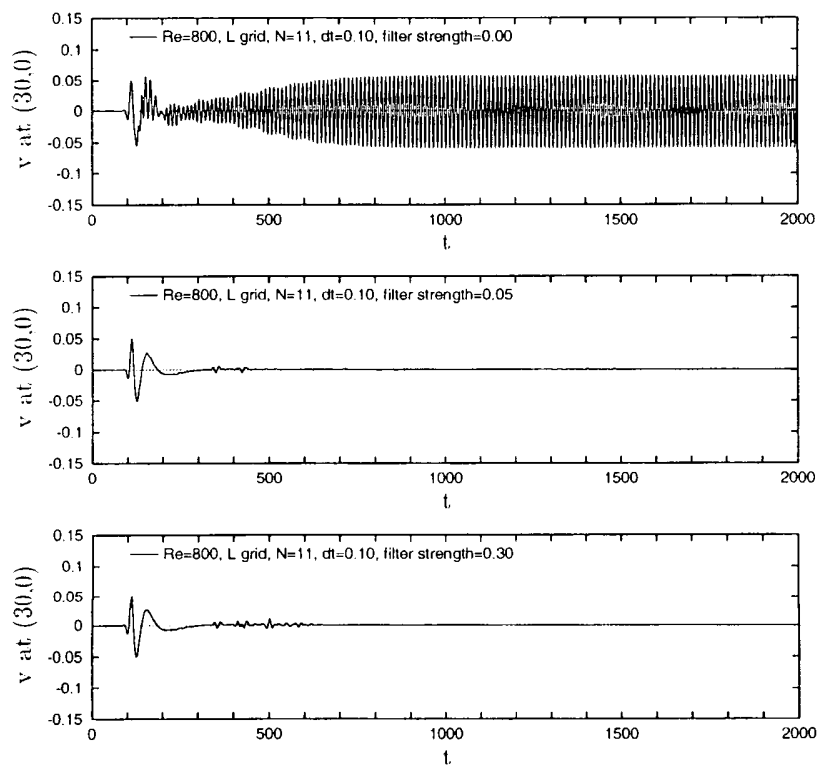

Figure 6.8. Comparison of the filtered with the un-filtered solutions of L811 with time step $d t=0.10$. Top: no filter; Middle: filter (filter strength 0.05); Bottom: filter (filter strength 0.3). 
behavior of existing numerical schemes. A third building block is to include nonlinear dynamics and bifurcation theories as an integral part of the numerical process whenever it is possible. A fourth building block is to construct appropriate adaptive spatial and temporal discretizations that are suitable for the underlying governing equation. The last building block is to construct appropriate adaptive numerical dissipation/filter controls for long time integrations, complex high speed turbulent and combustion simulations (Sjögreen \& Yee 2001, Yee \& Sjögreen 2001).

The need for the study of dynamics of numerics is prompted by the fact that the type of problem studied using CFD has changed dramatically over the past two decades. CFD is also undergoing an important transition, and it is increasingly used in nontraditional areas. But even within its field, many algorithms widely used in practical CFD applications were originally designed for much simpler problems, such as perfect or ideal gas flows. As can be seen in the literature, a straightforward application of these numerical methods to high speed flows, nonequilibrium flows, advanced turbulence modeling or combustion related problems can lead to wrong results, slow convergence, or even nonconvergent solutions. The need for new algorithms and/or modification and improvement to existing numerical methods in order to deal with emerging disciplines is evident. We believe the nonlinear dynamical approach for CFD can contribute to the success of this goal.

We have revealed some of the causes of spurious phenomena due to the numerics in an attempt to improve the understanding of the effects of numerical uncertainties in CFD. We have shown that guidelines developed using linearization methods are not always valid for nonlinear problems. We have gained an improved understanding of long time behavior of nonlinear problems and nonlinear stability, convergence, and reliability of time-marching approaches. We have learned that numerics can introduce and suppress chaos and can also introduce chaotic transients. The danger of relying on DNS to bracket closely the the onset of turbulence and chaos is evident.

We illustrated with practical CFD examples that exhibit properties and qualitative behavior similar to those of elementary examples in which the full dynamical behavior of the numerics can be analyzed. The observed spurious behavior related to under-resolved grid cases is particularly relevant to DNS and large Eddy simulation (LES). Spatial resolutions in DNS and LES are largely dictated by the computer power. These studies serve to point out the various possible dangers of misinterpreting numerical simulations of realistic complex flows that are constrained by available computing power.

As can be seen, recent advances in dynamics of numerics show the advantage of adaptive step size error control for long time integration of nonlinear 
ODEs. Although much research is needed to construct suitable yet practical similar adaptive methods for PDEs, these early developments lead our way to future theories. There remains the challenge of constructing practical spatial and temporal adaptive methods for time-accurate computations, and constructing adaptive step size control methods that are suitable yet practical for time marching to the steady state for aerospace CFD applications. Another even more challenging area is the quest for an adaptive numerical scheme that leads to guaranteed and rapid convergence to the correct steady-state numerical solutions.

\section{Acknowledgments}

The author wishes to thank her collaborators Peter Sweby, Laurence Keefe, John Torczynski, Ronald Henderson, Maxim Poliashenko, David Griffiths, Andre Lafon and Andrew Stuart, for contributing to her earlier work. The contributions of Section 6.2 by Laurence Keefe, Section 6.3 by Maxim Poliashenko, and Section 6.4 by Paul Fischer, John Torczynski and Ronald Henderson are gratefully acknowledged. Special thanks to Dennis Jespersen, Unmeel Mehta, Murray Tobak and Marcel Vinokur for their suggestions and critical review of the manuscript.

\section{References}

Brown, D.L. and Minion, M.L. (1995), "Performance of Under-resolved Two-Dimensional Incompressible Flow Simulations," J. Comput. Phys., Vol. 122, pp. 165-183.

Butcher, J.C. (1987), Numerical Analysis of Ordinary Differential Equations, John Wiley \& Son, Chichester.

Davidson, B. (1997), Large Scale Continuation and Numerical Bifurcation for PDE's, SIAM J. of Numer. Analy., Vol. 34, No. 5, pp. 2008-2027.

Doedel, E. (2000), AUTO: Software for Continuation and Bifurcation Problems in Ordinary Differential Equations, Concordia University, Montreal, Canada and Cal. Tech., Pasadena, Calif.

Ehrenstein, U. and Koch, W. (1991), "Nonlinear bifurcation study of plane Poiseuille flow," J. Fluid Mech., Vol. 228, pp. 111-148.

Fischer, P.F. and Mullen, J.S., (2001) "Filter-Based Stabilization of Spectral Element Methods", Comptes Rendus de l'Académie des sciences Paris, t. 332, Série I - Analyse numérique, 265-270 (2001).

Fischer, P.F., (2001) Private communication.

Fortin, A., Jardak, M., Gervais, J.J. and Pierre, R. (1996), "Localization of Hopf Bifurcations in Fluid Flow Problems," Intern. J. Numer. Meth. Fluids.

Gresho, P.M., Gartling, D.K., Torczynski, J.R., Cliffe, K.A., Winters, K.H., Garrett, T.J., Spencer, A. and Goodrich, J.W. (1993), "Is the Steady Viscous In- 
compressible Two-Dimensional Flow Over a Backward-Facing Step at $\mathrm{Re}=800$ Stable?" Intern. J. Numer. Meth. Fluids, Vol. 17, pp. 501-541.

Grebogi, C., Ott, E. and Yorke, J.A. (1983), "Crises, Sudden Changes in Chaotic Attractors, and Transient Chaos," Physica 7D, pp. 181-200.

Griffiths, D.F., Sweby, P.K. and Yee, H.C. (1992a), "On Spurious Asymptotes Numerical Solutions of Explicit Runge-Kutta Schemes," IMA J. Numer. Anal., Vol. 12, pp. 319-338.

Griffiths, D.F., Stuart, A.M. and Yee, H.C. (1992b), "Numerical Wave Propagation in Hyperbolic Problems with Nonlinear Source Terms," SIAM J. of Numer. Analy., Vol. 29, pp. 1244-1260.

Henderson, R. D., (1999) "Adaptive Spectral Element Methods for Turbulence and Transition, in High-Order Methods for Computational Physics", T. J. Barth and H. Deconinck, editors. Springer.

Herbert, Th. (1976), Lecture Notes in Physics 59, Springer-Verlag, p235.

Hoppensteadt, F.C. (1993), Analysis and Simulation of Chaotic Systems, Springer-Verlag, New York.

Kaiktsis, L., Karniadakis, G.E. and Orszag, S.A. (1991), "Onset of ThreeDimensionality, Equilibria, and Early Transition in Flow Over a BackwardFacing Step," J. Fluid Mech., Vol. 231, pp. 501-528.

Kaiktsis, L., Karniadakis, G.E. and Orszag, S.A. (1996), "Unsteadiness and Convective Instabilities in Two-Dimensional Flow Over a Backward-Facing Step," J. Fluid Mech., Vol. 321, pp. 157-187.

Keefe, L., Moin, P. and Kim, J. (1992), "The Dimension of Attractors Underlying Periodic Turbulent Poiseuille Flow," J. Fluid Mech., Vol. 242, pp. 1-29.

Keefe, L. (1988-1996), unpublished; private communication.

Kim, J., Moin, P. and Moser, R. (1987), "Turbulence statistics in fully developed channel flow at low Reynolds number,' J. Fluid Mech., Vol. 177, pp. 133-166.

Keller, H.B. (1977), "Numerical Solution of Bifurcation and Nonlinear Eigenvalue Problems," Applications of Bifurcation Theory, P.H. Rabinowitz, ed., Academic Press, pp. 359-384.

Lafon, A. and Yee, H.C. (1991), "Dynamical Approach Study of Spurious Steady-State Numerical Solutions for Nonlinear Differential Equations, Part III: The Effects of Nonlinear Source Terms in Reaction-Convection Equations," NASA Technical Memorandum 103877, July 1991; International J. Comput. Fluid Dyn., Vol. 6, pp. 1-36, 1996.

Lafon, A. and Yee, H.C. (1992), "Dynamical Approach Study of Spurious Steady-State Numerical Solutions of Nonlinear Differential Equations, Part IV: Stability vs. Numerical Treatment of Nonlinear Source Terms," ONERA-CERT 
Technical Report DERAT 45/5005.38, also, International J. Comput. Fluid Dyn., Vol. 6, pp. 89-123, 1996.

LeVeque, R.J. and Yee, H.C. (1990), "A Study of Numerical Methods for Hyperbolic Conservation Laws with Stiff Source Terms," J. Comput. Phys., Vol. 86, pp. 187-210.

Maday, Y. and Patera, A.T., (1989) "Spectral Element Methods for the Navier-Stokes Equations", State of the Art Survey in Computational Mechanics, A.K. Noor, ed., ASME, New York, pp. 71-143.

Maday, Y., Patera, T. and Rønquist, E.M., (1990) "An operator-integrationfactor splitting method for time-dependent problems: Application to incompressible fluid flow. newblock J.Sci.Comput., Vol. 5, pp. 263-292.

Minion, M.L. and Brown, D.L., (1997) "Performance of Under-resolved Two-Dimensional Incompressible Flow Simulations II", J. Comput. Phys. 138, 734-765.

Moin, P., and Kim, J. (1982), "Numerical investigation of turbulent channel flow," J. Fluid Mech., Vol. 118, pp. 341-378.

Moore, D.R., Weiss, N.O. and Wilkins, J.M. (1990), "The Reliability of Numerical Experiments: Transitions to Chaos in Thermosolutal Convection," Nonlinearity, Vol. 3, pp. 997-1014.

Moretti, G. and Abbett, M., (1966), "A Time-Dependent Computational Method for Blunt Body Flows,” AIAA Journal, Vol. 4, pp. 2136-2141.

NEKTON User's Guide, Version 2.8, 1991, Nektonics Inc., Cambridge, MA.

Orszag, S. (1971), "Accurate solution of the Orr-Sommerfeld stability equation," J. Fluid Mech., Vol. 50, pp. 689-703.

Poliashenko, M. and Aidun, C.K. (1995), "Computational Dynamics of Ordinary Differential Equations," Intern. J. Bifurcation and Chaos, Vol. 5, pp. 159-174.

Schreiber, R. and Keller, H.B. (1983), "Spurious Solution in Driven Cavity Calculations," J. Comput. Phys., Vol. 49, pp. 310-333.

Shroff, G.M. and Keller, H.B. (1993), Stabilisation of unstable procedures: The RPM, SIAM J. of Numer. Analy., Vol. 30, No. 4, pp. 1099-1120.

Shubin, G.R., Stephens, A.B. and Glaz, H.M. (1981), "Steady Shock Tracking and Newton's Method Applied to One-Dimensional Duct Flow,' J. Comput. Phys., Vol. 39, pp. 364-374.

Sjögreen, B. and Yee, H.C., (2000) "Multiresolution Wavelet Based Adaptive Numerical Dissipation Control for Shock-Turbulence Computations", RIACS Report 01.01, NASA Ames research center (Oct 2000).

Sjögreen, B. and Yee, H.C., (2001) "On Entropy Splitting, Linear and Nonlinear Numerical Dissipations and Long-Time Integrations, Proceedings of the 
5th Internat. Conf. on Spectral and High Order Methods, Uppsala, Sweden, June 11-15, 2001.

Spalart, P.R., Moser, R.D. and Rogers, M.M. (1991), "Spectral methods for the Navier-Stokes equations with one infinite and two periodic directions," J. Comput. Phys., Vol. 96, p297.

Stephens, A.B. and Shubin, G.R. (1981) "Multiple Solutions and Bifurcation of Finite Difference Approximations to Some Steady Problems of Fluid Dynamics," SIAM J. Sci. Statist Comput., Vol. 2, pp. 404-415.

Sweby, P.K. and Yee, H.C. (1994), "On the Dynamics of Some Grid Adaptation Schemes," Proceedings of the 4th International Conference on Numerical Grid Generation in CFD and Related Fields, University College of Swansea, UK, also RIACS Technical Report 94.02, Feb. 1994.

Sweby, P.K., Lafon, A. and Yee, H.C. (1995), "On the Dynamics of Computing a Chemically Relaxed Nonequilibrium Flow," presented at the ICFD Conference on Numerical Methods for Fluid Dynamics, April 3-6, 1995, Oxford, UK.

Thompson, J.M.T. and Stewart, H.B. (1986), Nonlinear Dynamics and Chaos, John Wiley, New York.

Torczynski, J.R. (1993), "A Grid Refinement Study of Two-Dimensional Transient Flow Over a Backward-Facing Step Using a Spectral-Element Method," FED-Vol. 149, Separated Flows, ASME 1993, J.C. Dutton and L.P. Purtell, editors.

Yee, H.C., Sweby, P.K. and Griffiths, D.F. (1991), "Dynamical Approach Study of Spurious Steady-State Numerical Solutions for Nonlinear Differential Equations, Part I: The Dynamics of Time Discretizations and Its Implications for Algorithm Development in Computational Fluid Dynamics," NASA TM102820, April 1990; J. Comput. Phys., Vol. 97, pp. 249-310.

Yee, H.C. and Sweby, P.K. (1992), "Dynamical Approach Study of Spurious Steady-State Numerical Solutions for Nonlinear Differential Equations, Part II: Global Asymptotic Behavior of Time Discretizations," RNR-92-008, March 1992, NASA Ames Research Center; also International J. Comput. Fluid Dyn., Vol. 4, pp. 219-283, 1995.

Yee, H.C. and Sweby, P.K. (1993), "Global Asymptotic Behavior of Iterative Implicit Schemes," RIACS Technical Report 93.11, December 1993, NASA Ames Research Center, also Intern. J. Bifurcation \& Chaos, Vol. 4, pp. 1579-1611.

Yee, H.C. and Sweby, P.K. (1995), "On Super-Stable Implicit Methods and Time-Marching Approaches," RIACS Technical Report 95.12, NASA Ames Research Center, July 1995; also, Proceedings of the Conference on Numerical Methods for Euler and Navier-Stokes Equations, Sept. 14-16, 1995, University 
of Montreal, Canada; International J. Comput. Fluid Dyn. Vol. 8, pp. 265-286, 1997.

Yee, H.C. and Sweby, P.K. (1996), "Some Aspects of Numerical Uncertainties in Time Marching to Steady-State Computations," AIAA-96-2052, 27th AIAA Fluid Dynamics Conference, June 18-20, 1996, New Orleans, LA., AIAA J., Vol. 36, No. 5, pp. 712-724, 1998

Yee, H.C., Torczynski, J.R., Morton, S.A., Visbal, M.R. and Sweby, P.K. (1997), "On Spurious Behavior of CFD Simulations," AIAA 97-1869, Proceedings of the 13th AIAA Computational Fluid Dynamics Conference, June 29 - July 2, 1997, Snowmass, CO.; also International J. Num. Meth. Fluids, Vol. 30, pp. 675-711, 1999.

Yee, H.C. and Sweby, P.K. (1997), "Dynamics of Numerics \& Spurious Behaviors in CFD Computations," Keynote paper, 7th ISCFD Conference, Sept. 15-19, 1997, Beijing, China, RIACS Technical Report 97.06, June 1997.

Yee, H.C., Sandham, N.D. and Djomehri, M.J., (1999) ' 'Low Dissipative High Order Shock-Capturing Methods Using Characteristic-Based Filters", J. Comput. Phys., 150 199-238.

Yee, H.C., Vinokur, M. and Djomehri, M.J., (1999) "Entropy Splitting and Numerical Dissipation," NASA Technical Memorandum 208793, August, 1999, NASA Ames Research Center; J. Comput. Phys., 162, 33 (2000).

Yee, H.C. and Sjögreen, B., (2001a) "Adaptive Numerical-Dissipation/Filter Controls for High Order Numerical Methods," Proceedings of the 3rd International Conference on DNS/LES, Arlington, Texas, August 4-9, 2001.

Yee, H.C. and Sjögreen, B., (2001b) "Designing Adaptive Low Dissipative High Order Schemes for Long-Time Integrations," Turbulent Flow Computation, (Eds. D. Drikakis \& B. Geurts), Kluwer Academic Publisher; also RIACS Technical Report, Dec. 2001. 